\title{
Circulation et ancrage local des bouffons maliens : morphogenèse d'un groupe social
}

\section{Laure Carbonnel}

\section{OpenEdition}

Journals

Édition électronique

URL : https://journals.openedition.org/e-migrinter/258

DOI : 10.4000/e-migrinter.258

ISSN : 1961-9685

Éditeur

UMR 7301 - Migrinter

Édition imprimée

Date de publication : 1 septembre 2013

Pagination : 65-77

ISSN : 1961-9685

\section{Référence électronique}

Laure Carbonnel, «Circulation et ancrage local des bouffons maliens : morphogenèse d'un groupe social », e-Migrinter [En ligne], 11 | 2013, mis en ligne le , consulté le 20 mai 2021. URL : http:// journals.openedition.org/e-migrinter/258; DOI : https://doi.org/10.4000/e-migrinter.258 


\section{Circulation et ancrage local des bouffons maliens : morphogenèse d'un groupe social*}

\section{Laure Carbonnel}

$\mathbf{L}$

a bouffonnerie se caractérise par des danses et des comportements burlesques qui se manifestent en particulier au cours de cérémonies rituelles. Les études qui lui sont consacrées ${ }^{1}$ sont peu coutumières des problématiques de la circulation car elles se focalisent avant tout sur la signification et les incidences sociales de ces comportements comiques et transgressifs tels qu'ils apparaissent de manière sporadique dans un contexte social donné. Mes recherches $^{2}$ interrogent moins le sens que les conditions de réalisation de la bouffonnerie malienne (Koroduga en bambara); non seulement en tant que pratique, mais également en tant qu'activité exercée par un groupe social particulier composé d'hommes et de femmes. Partant des individus et de leurs réseaux de relations, j'ai suivi le déroulement dans le temps des prestations bouffonnes et observé les modalités de leurs organisations sociales.

\footnotetext{
*Je remercie les organisateurs et les participants des journées «Et l'immobilité dans la circulation? », ainsi que Sylvie Beaud, Azita Bathaie et Mickaële Lantin Mallet pour leurs commentaires.

${ }^{1}$ Parmi lesquels: Pâques (1954) Zahan (1960), Dieterlen \& Cissé (1972), Luneau (1974), Colleyn (1996, 2009) pour le Mali, ainsi que Handelman (1981), De Sales (1995), Tarabout (1998) pour l'Amérique du Sud, la Mongolie et l'Inde. La question de la circulation est présente dans l'étude d'Eric Jolly (1994) mais elle porte moins sur les individus que sur les autels pour la formation de nouvelles sociétés initiatiques.

${ }^{2}$ Les matériaux et les analyses mobilisés dans cet article sont issus d'un travail de recherche doctorale en cours de réalisation.
}

Appelés à intervenir au sein de cérémonies rituelles (mariages, circoncisions, etc.) ou séculières (inaugurations, fêtes nationales, festivals, etc.), les bouffons se rassemblent pour se rendre d'un village à l'autre ou d'un quartier à l'autre. La circulation comprise ici comme un déplacement pour l'exercice d'une activité se trouve donc au fondement de la pratique de la bouffonnerie. Dans le même temps, les bouffons s'appuient sur un ancrage local défini ici comme l'inscription d'un individu ou d'une pratique au sein d'une communauté villageoise ou urbaine donnée ${ }^{3}$. Qu'est-ce qui est mis en œuvre dans ces deux dimensions et comment s'articulentelles?

Dans un premier temps, je montrerai de quelle manière l'articulation entre ancrage local et circulation participe à la reproduction et à l'organisation sociale de ce groupe. Tout d'abord en analysant l'implication de l'activité de bouffonnerie qui, par sa mobilité, assure la visibilité de la catégorie sociale koroduga; puis en partant des regroupements bouffons dans l'espace villageois, dont l'organisation s'inscrit dans la circulation. Dans un second temps, j'interrogerai plus précisément les

\footnotetext{
${ }^{3} \mathrm{Au}$ Mali, les Bambaras, où qu'ils vivent, font référence au village de leur père ou de leur grand-père pour désigner leurs localités d'origine. La résidence n'est donc pas l'unique critère pour définir la localité d'appartenance d'un individu. D'une manière générale, un étranger venu s'installer dans un village donné pourra, selon la position qu'il y acquiert, avoir des difficultés à participer à la vie sociale et économique du village (avec un accès limité aux champs, par exemple) et tendre à être exclu des décisions politiques. Plusieurs facteurs viennent complexifier cette notion d'ancrage local. Dans cette communication, les cas étudiés restent au niveau le plus simple, celui d'anciennes familles villageoises résidant dans leur localité d'origine.
} 
mécanismes mis en œuvre dans une pratique de bouffonnerie qui confronte des membres mobiles et des membres immobiles. J'analyserai tour à tour les effets de l'immobilité sur la circulation et de la circulation sur l'immobilité en dégageant les configurations relationnelles particulières ainsi générées.

\section{L'articulation entre ancrage local et circulation dans l'organisation sociale des bouffons}

\section{La reproduction sociale du groupe s'appuie sur une activité impliquant une mobilité intervillageoise}

Koroduga est une catégorie qui, à l'image des groupes statutaires (griots, forgerons), rattache une activité à un groupe. Elle ne désigne pas seulement une pratique (la bouffonnerie); elle désigne avant tout une identité sociale qui se transmet soit par la filiation agnatique (par le père), soit par l'adoption. Le groupe de pairs forgé par cette appartenance commune comprend ainsi à la fois des individus pratiquant la bouffonnerie (les actifs) et des non pratiquants (les inactifs). Bien qu'elle ne conditionne pas l'appartenance à la catégorie sociale, l'activité joue tout de même un rôle central dans la reproduction du groupe social.

Tout d'abord, contrairement aux identités sociales ethniques ou de statut qui constituent au Mali les deux principaux systèmes de catégorisation des individus, l'identité de bouffon reste invisible tant qu'elle n'est pas manifestée explicitement. Il n'existe pas de patronymes qui lui soient associés et elle n'apparait pas dans le système des relations à plaisanterie qui, au Mali, permet de rappeler quotidiennement, dans les interactions ordinaires, certaines des catégories d'appartenances des individus ${ }^{4}$.

${ }^{4}$ Les bouffons participent aux relations à plaisanterie mais par l'intermédiaire de leurs identifications ethniques ou de caste.
Au-delà du réseau d'interconnaissance (dans un village les familles korodugaw sont connues), l'activité constitue donc son principal mode de visibilité. Selon mes interlocuteurs, la perte de visibilité de leur groupe met en péril son existence puisqu'ils affirment qu'ils étaient sur le point de disparaitre : Il faut que les gens nous connaissent, ce qui passe en particulier par l'exercice de leur activité.

Les raisons évoquées pour expliquer l'abandon de ces pratiques, voire le reniement de cette identité, sont l'islamisation et la modernité qui s'opposeraient à la korodugaya présentée comme une coutume ancienne et associée aux pratiques animistes. Elles ne s'opposent pas d'un point de vue idéologique strict puisque l'islamisation n'est pas récente ${ }^{5}$ et coexiste depuis des siècles avec l'animisme (Kassibo, 1992) et, de surcroît, les korodugaw rencontrés sont pour une grande majorité de confession musulmane. Le recul de la bouffonnerie semble avant tout lié aux modifications des espaces de pratiques cultuelles. L'islam s'est peu à peu imposé de manière exclusive dans l'espace public villageois et urbain face à des pratiques cérémonielles animistes accomplies notamment par les sociétés initiatiques dans lesquelles les korodugaw prenaient une part importante. De grandes cérémonies villageoises ont toujours lieu (circoncisions, intronisation de chefs de village, cultes effectués pour conjurer une mauvaise saison des pluies, etc.), mais elles apparaissent plus conjoncturelles que routinières et semblent moins centrales dans l'organisation sociale locale. Avec l'abandon de ces manifestations publiques et collectives dans la région de Ségou, les korodugaw se voient retirer un espace d'intervention et une légitimité à l'échelle villageoise. Les cadres sociaux étant moins favorables à la pratique de la bouffonnerie, le nombre de bouffons actifs a

${ }^{5}$ L'islamisation a été portée par des représentants politiques dès le XIV ${ }^{e}$ siècle, en particulier l'empereur Mansa Moussa et s'est imposée avec Samori Toure qui a instauré une théocratie au XIX ${ }^{\mathrm{e}}$ siècle. 
diminué $^{6}:$ des villages autrefois réputés pour les connaissances et l'activité des korodugaw ne comptent plus aucun membre actif. Néanmoins, ces dernières années, la tendance semble s'inverser grâce notamment au développement des associations et des manifestations bouffonnes intervillageoises comme le suggèrent les propos d'un de mes interlocuteurs, le chef bouffon d'un village : Maintenant que nous sommes une vraie association et que nous nous promenons beaucoup, les jeunes nous voient et se présentent d'eux-mêmes. L'association qu'il évoque est une association de loi 1901 basée dans la ville de Ségou qui regroupe des bouffons provenant de villages s'étendant sur un rayon d'une cinquantaine de kilomètres ${ }^{7}$.

Les modalités de regroupements et les participations cérémonielles observées entre 2006 et 2010 au Mali témoignent de l'importance de la mobilité intervillageoise ${ }^{8}$ : Un koroduga seul ne peut faire une festivité bouffonne affirment-ils. Or, bien souvent, un village ne compte pas suffisamment de bouffons actifs pour participer à une cérémonie donnée; c'est pourquoi les membres ou les groupements de différentes localités doivent se rassembler. Cette mobilité intervillageoise concerne également les occasions cérémonielles elles-mêmes, en particulier celles organisées par les «hommes de pouvoir» (faamaw) avec qui les bouffons entretiennent des relations de réciprocité. Elles tendent à se multiplier

\footnotetext{
${ }^{6} \mathrm{La}$ tendance est à l'exclusivité religieuse. L'islam, religion des dominants, constitue un vecteur de promotion sociale pour les nouveaux convertis et se présente comme l'avenir opposé aux pratiques cultuelles passéistes. Pour autant, l'islam continue encore aujourd'hui à coexister avec les pratiques animistes qui, loin de s'opposer à la modernité, l'accompagnent: méprisés en public, les détenteurs de ces connaissances sont particulièrement sollicités dans leur village.

${ }^{7}$ L'un des regroupements intervillageois associé est plus ancien, il date du début des années 1990 mais l'association ségouvienne semble avoir accéléré ce processus.

${ }^{8}$ Cela apparait clairement dans leurs processions qui prennent une forme différente en milieu citadin et en milieu villageois.
}

depuis la décentralisation de 1996 qui a conduit à la création de communes rurales regroupant plusieurs villages. Suivant les évolutions de l'espace politique, les bouffons participent aux cérémonies organisées par les maires de ces communes rurales, trouvant ainsi de nouvelles opportunités d'intervention. Enfin, les bouffons euxmêmes mettent en œuvre de nouvelles pratiques cérémonielles telles les baptêmes des enfants qui leur sont «donnés». L'adoption n'est pas un fait nouveau puisqu'une femme dont les enfants décèdent en bas âge peut tenter de rompre ce cycle en offrant à son nouveau-né une nouvelle identité sociale, comme celle de koroduga. L'enfant reste dans sa famille, mais sa mère et lui reçoivent leurs médecines et leurs protections face aux sorciers considérés comme étant à l'origine des décès. Le processus d'adoption se poursuit par l'organisation d'une festivité bouffonne qui se déroule fréquemment le jour de la circoncision de l'enfant. Avec le développement d'associations intervillageoises de korodugaw, des cérémonies de baptêmes sont organisées indépendamment des rites de passage de l'enfant. Les korodugaw ne se contentent plus de répondre à une invitation, ils choisissent de réaliser ces baptêmes de manière autonome grâce aux réseaux de relations bouffons qu'ils consolident dans le même temps.

En résumé, cette catégorie sociale qui regroupe aussi bien des membres pratiquant la bouffonnerie que des non pratiquants doit s'appuyer sur une activité et des pratiques sans cesse réactivées pour que l'identité sociale koroduga se maintienne de manière visible dans l'espace public. La diminution $\mathrm{du}$ nombre d'actifs et d'occasions cérémonielles renforce le caractère intervillageois des cérémonies bouffonnes inscrivant leur activité dans la mobilité. Les déplacements des bouffons s'appuient néanmoins dans le même temps sur un ancrage local; une articulation qui apparaitt clairement dans leurs modalités d'organisation. 


\section{Des groupements villageois aux réseaux}

Par naissance ou par adoption, des individus sont identifiés comme appartenant à la catégorie sociale koroduga. Il s'agit d'une identité sociale portée par des hommes et des femmes, qu'ils pratiquent ou non la bouffonnerie. Elle s'exprime dans l'organisation villageoise par l'intermédiaire d'un chef local qui se trouve ainsi à la tête d'un groupe social koroduga villageois. Fondé sur le seul partage d'une identité commune, ce groupement est composé de l'ensemble des familles koroduga d'une même localité. Certains de ces membres actifs se distinguent néanmoins en se regroupant pour participer à des cérémonies bouffonnes dans d'autres localités. Ils forment alors un second type d'unité sociale, le groupement d'activité. Fondé cette fois sur l'activité de bouffonnerie et les déplacements en dehors du village d'origine, il rassemble majoritairement des hommes. En effet, les déplacements cérémoniels des femmes actives en dehors du village sont le plus souvent conditionnés par des relations de parenté préalables qui les lient avec certains habitants de la localité visitée, ce qui limite leur mobilité intervillageoise pour la pratique de la bouffonnerie. Ce n'est pas le cas pour les hommes, mais leur mobilité est tout de même restreinte par d'autres contraintes: Tout le monde ne peut pas se déplacer, il faut bien que certains restent au village pour s'occuper de la maison, des champs, du bétail. Ainsi, parmi les familles favorables à cette pratique, un seul homme par fratrie intègre usuellement un groupement d'activité. Lorsqu'un seul homme dans le village s'adonne à ces déplacements cérémoniels bouffons, il rejoint alors un groupement d'activité d'un autre village.

Les deux types de groupements bouffons - villageois et d'activités - sont ancrés localement, dans le sens où leurs représentants sont attachés à un village

${ }^{9}$ Les migrations des villageois partant dans les centres urbains pour travailler suivent également une division des rôles et de la mobilité au sein d'une fratrie. particulier, suivant en cela un principe d'affiliation spatial commun au Mali ${ }^{10}$ C'est la différence de mobilité entre ces membres qui introduit ensuite une distinction entre les groupements mais aussi une hiérarchisation entre les individus: Elle, c'est une vraie koroduga, elle se promène beaucoup! Comme le suggère ce propos émanant d'un chef koroduga, la mobilité intervillageoise des bouffons pour l'exercice de leur activité est fortement valorisée, par leurs pairs notamment, car elle participe à la propagation de ce qu'ils considèrent comme leur coutume. Ils distinguent alors clairement les immobiles restant dans l'enceinte villageoise des mobiles qui en sortent.

Lorsque ces derniers se rendent dans une autre localité, ils se séparent des korodugaw qui restent chez eux, mais rejoignent ceux qui résident dans le village visité. C'est pourquoi, au final, tous les bouffons sur le passage des mobiles sont concernés par leur circulation. En effet, les bouffons se rendent principalement dans les villages où se trouve un chef koroduga local ${ }^{11}$. En tant que représentant villageois des korodugaw, le chef local est informé et sollicité par les autorités afin de mobiliser les membres de son groupe au cours d'une manifestation pour laquelle il peut également appeler des bouffons étrangers au village. Pour ces derniers, il est le représentant local, l'hôte et le référent incontournable pour participer ou initier une cérémonie dans sa localité. Il participe ainsi activement à la circulation de ses pairs. Plus encore, parmi les bouffons «immobiles » à l'échelle intervillageoise, se trouvent des hommes et majoritairement des femmes qui circulent dans leur localité

${ }^{10}$ Malgré la mobilité résidentielle des individus et des familles, les Bambaras présentent habituellement leur village d'origine qui est celui de leur père ou de leur grand-père.

${ }^{11}$ Tous les villages ne comportent pas de membres de cette catégorie sociale, et encore moins de chef koroduga local dont la présence implique une reconnaissance et une acceptation par les autorités locales (le chef du village). 
Figure $\mathrm{n}^{\circ} \mathbf{1}$ : les organisations bouffonnes villageoises mobilisées dans les déplacements cérémoniels.

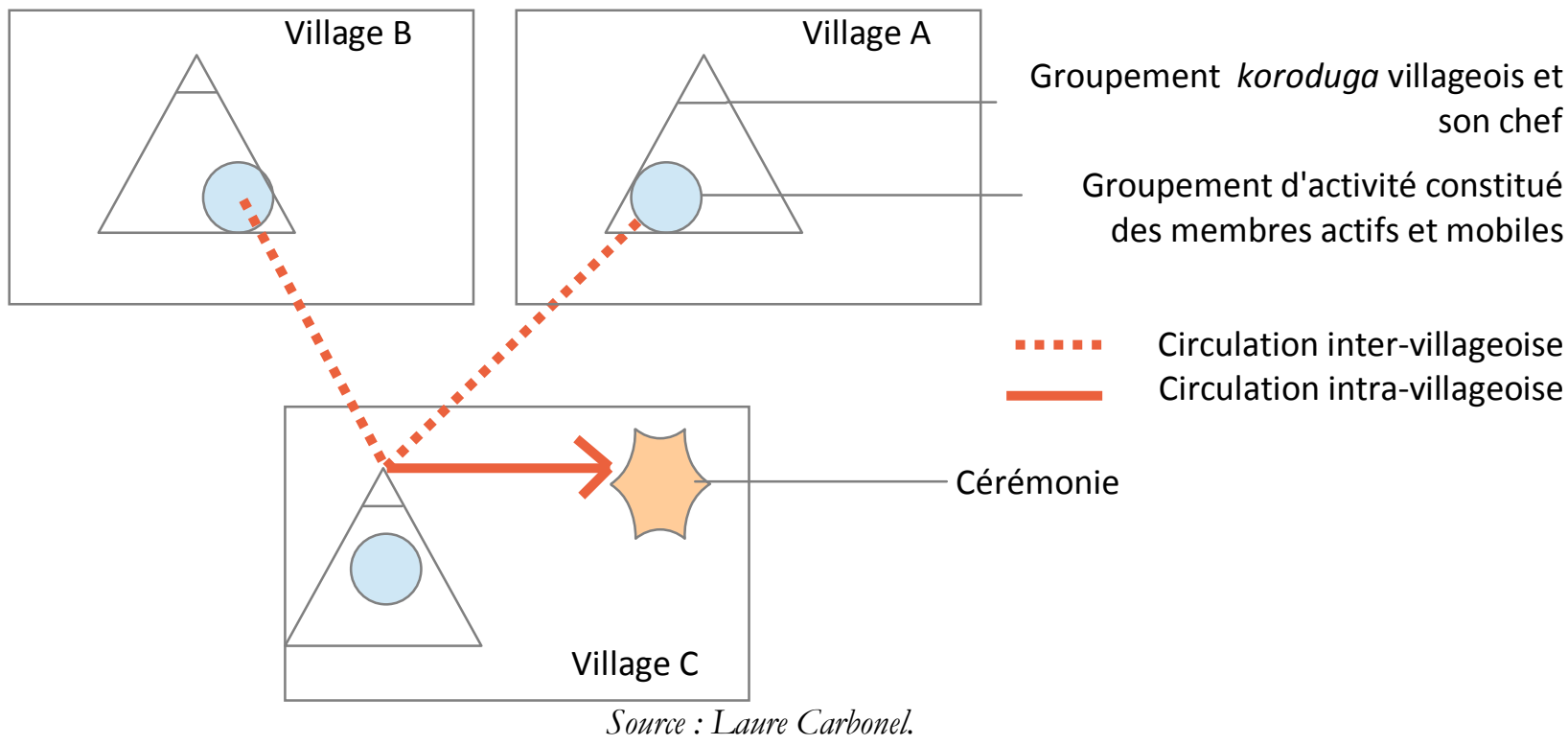

pour pratiquer la bouffonnerie. Ces deux positions sont donc relatives: d'une part, car les immobiles participent à la circulation; d'autre part, car elles dépendent de l'échelle spatio-temporelle considérée, intervillageoise, ou villageoise (figure 1).

Non seulement la circulation concerne ces deux types de regroupements et de mobilités, mais plus encore, elle les englobe en forgeant un réseau de relations.

Un koroduga actif est appelé à participer à une cérémonie grâce à son réseau de connaissances (dans ou hors du champ de la bouffonnerie). Il rassemble alors autour de lui les membres de son groupe d'activité ou au-delà si l'ampleur de la manifestation le permet, pour se rendre dans la localité où se déroulent les festivités. Avant d'entrer dans le village, ces étrangers préviennent le chef koroduga local et rejoignent les membres du groupement koroduga villageois pour participer ensemble à la cérémonie et pour rendre visite aux habitants et aux bouffons inactifs. L'ensemble des bouffons - actifs, inactifs, mobiles, immobiles, locaux, étrangers - est mis en relation dans la circulation qui actualise leur identité sociale commune audelà des différences de pratiques et de localité qui les distingue. Chacun mettant en relation les différents membres qu'il connait selon le lieu où il se trouve, ce réseau n'a pas de limites, il se fonde sur des liens qui évoluent au fil du temps. Autrement dit, ce réseau se forge dans une dynamique de circulation qui s'appuie sur l'ancrage local de ces membres.

\section{S'ancrer localement pour circuler}

\section{La mobilité intervillageoise : des étrangers dans une localité}

L'activité de bouffonnerie conduisant les korodugaw à se rendre d'une localité à l'autre, le couple mobile/immobile s'exprime dans une relation entre étrangers au village et locaux. Toute personne qui n'est pas originaire de la localité dans laquelle elle se trouve est considérée comme étrangère, qu'elle y réside ou non. Cette extériorité est accentuée par le fait que la majorité des bouffons de passage n'ont pas de liens spécifiques avec des habitants du village, ils ne sont pas connus personnellement (leurs noms de famille, leurs autres identités 
sociales, etc.). Leurs déplacements en groupes et leurs parures permettant aisément d'identifier leur identité sociale de koroduga, elle pourrait suffire à légitimer leur présence, puisque chacun sait que les bouffons participent à des cérémonies, qu'ils se rendent d'un village à l'autre, qu'ils ont des comportements transgressifs et qu'ils sollicitent des dons. Cependant, contrairement aux membres d'autres catégories sociales ${ }^{12}$ se déplaçant de village en village, les bouffons fournissent aux habitants des informations supplémentaires permettant de les situer. Avant que les festivités bouffonnes ne commencent, l'un des chefs koroduga en visite s'adresse aux spectateurs rassemblés pour présenter les localités d'origine des membres présents : les gens de tels villages ou de telles régions sont venus chez vous. Aussi, ce même porte-parole annonce-t-il dans la foulée la raison de leur présence: participer à une cérémonie ou initier des festivités bouffonnes (pour rendre hommage à un membre défunt ou rendre visite aux enfants donnés).

Leurs déplacements sont donc toujours orientés vers une finalité clairement énoncée, respectant ainsi une règle de bienséance malienne qui s'applique particulièrement aux étrangers ${ }^{13}$. Ces bouffons en déplacement ont également un référent dans le village visité en la personne du chef de groupe koroduga local qui les reçoit, leur trouve un hébergement et organise leurs repas. Enfin, ils se mettent sous l'autorité du chef du village auquel ils rendent visite à leur arrivée afin qu'il les autorise à poursuivre la procession dans les

\footnotetext{
${ }^{12} \mathrm{En}$ particulier les gaoulow, présentés par mes interlocuteurs korodugaw et dans la littérature (Luneau, 1974) comme des mendiants qui se déplacent et s'installent chez des villageois quels qu'ils soient, dans le seul but de recevoir des dons et sans expliciter leur origine.

${ }^{13}$ Lorsqu'un étranger arrive dans une localité et se rend chez un habitant, la première question qui lui est posée, après son nom porte sur sa localité d'origine, puis il doit annoncer son intention, la raison de sa venue. Ensuite, son logeur doit l'accompagner auprès des autorités locales (chef de village, préfet), pour le présenter.
}

ruelles et les maisons. Leurs déplacements font donc l'objet d'un triple cadrage : d'où ils viennent (leur localité d'origine), pour quelles raisons ils sont venus (leur intention), qui est le villageois référent (leur point d'ancrage dans le village visité).

Les bouffons étrangers sont ancrés localement, mais dans le même temps leur pratique prend forme et sens dans la circulation. En route vers la localité où ils sont attendus, certains en moto, d'autres en vélo, ils effectuent de multiples arrêts dans les villages intermédiaires pour attendre le reste du groupe, pour boire, demander leur chemin, rendre visite à un proche, solliciter des dons, etc. Ces moments sont l'occasion d'interagir avec les passants, de se présenter à eux en tant que koroduga, de pratiquer la bouffonnerie par des jeux ou des pas de danse esquissés au son des percussions. De même, à leur arrivée dans la localité où se déroulent les festivités auxquelles ils entendent participer, leur intervention débute par une procession qui part de l'entrée du village ou de la concession d'accueil des étrangers, pour se rendre jusqu'à l'espace cérémoniel où ils effectuent leurs prestations dansées. Cette circulation en procession se distingue alors de déplacements qui auraient pour seule fonction utilitaire de se rendre d'un point à un autre. En effet, lors de certaines cérémonies, urbaines en particulier, les bouffons de différentes maisons et quartiers se rassemblent pour se rendre sur le lieu des festivités, mais ce n'est qu'une fois sur place qu'ils revêtent leurs parures de bouffon et qu'ils entament les chants, les musiques et les jeux bouffons. Dans ce cas, les déplacements n'ont d'autre finalité que celle d'arriver sur le lieu des festivités.

Lorsque, en revanche, ils se rendent dans une autre localité, qu'ils y passent la journée et bien souvent la nuit, et que leur participation cérémonielle n'est pas rémunérée par les organisateurs, les déplacements prennent la forme d'une procession qui précède mais également suit 
leur intervention cérémonielle. Au cours de ces processions (intra ou inter villageoises), ils prennent la peine de revêtir leurs parures, de faire résonner leurs percussions, d'entonner les chants bouffons de manière à ce qu'ils soient entendus et compris par les habitants. Ils donnent à voir publiquement leur identité sociale de koroduga, non pas seulement en participant à une cérémonie où ils sont attendus, mais en venant à la rencontre de l'ensemble des villageois. Ceux qu'ils croisent dans les ruelles, près des échoppes et devant lesquels ils s'arrêtent pour entamer une danse en cercle, ceux chez qui ils se rendent, ceux qui les regardent à l'entrée de la cour de leur maison ou pardessus le muret d'enceinte. Ces processions sont également l'occasion de susciter des dons auprès des habitants.

Ancrage local et circulation s'articulent alors à chaque étape de leur parcours. Lorsque les bouffons sont dans le village visité, ils s'inscrivent dans un espace en se mettant sous l'autorité du chef de village à qui ils demandent l'autorisation de circuler, puis ils entament leur procession. À l'inverse, sur le chemin qui les mène à cette localité, ils sont en cours de déplacement, tout en affirmant leurs différents ancrages locaux de départ et d'arrivée, lorsqu'ils explicitent d'où ils viennent et où ils vont. De cette articulation entre circulation et localisation nait une nouvelle dimension: l'appropriation de l'espace parcouru. Par leur manifestation publique, sonore et visuelle, ils exposent leur identité sociale: par leur intrusion dans les maisons et dans les ruelles, barrant momentanément le passage de ceux qui passent, interrompant les discussions et les activités ils s'imposent aux habitants. Ils impriment des normes vestimentaires et comportementales qui sont usuellement réprimées dans les interactions ordinaires entre adultes étrangers : parler publiquement de l'intimité (sexualité, scatologie), entrer dans les cuisines, se servir à manger, courir après les poulets, etc. L'absence de honte, au sens social de l'évitement et de la déférence, est de règle lorsqu'ils sont en représentation.
Cette appropriation de l'espace par la circulation s'effectue toujours en cherchant l'adhésion des habitants, par l'autorisation de circuler donnée par le chef du village, et par les dons des habitants qui attestent de la reconnaissance de leur statut. Cette inscription dans l'espace social villageois, qui se manifeste dans le chant Le village est au chef de village, les ruelles sont à nous, s'appuie également sur la participation des bouffons résidents.

\section{Les bouffons locaux et leurs rôles dans la circulation}

Les bouffons résidents vont faciliter la circulation de ces étrangers, tout d'abord en leur donnant l'accès à la localité, ce qui est avant tout le rôle des chefs korodugaw locaux. Ce n'est pas seulement le respect de l'ordre de préséance qui les place comme premiers interlocuteurs des étrangers. Dans la ville de Ségou, lors de l'inauguration d'une nouvelle mosquée, le chef koroduga souhaitant accueillir par ces chants et ces danses l'arrivée du président malien avait pris place à l'intérieur du cordon de sécurité formé par des gendarmes et des policiers. À l'extérieur, parmi la population rassemblée figuraient d'autres bouffons. Voyant leur représentant à l'entrée de la mosquée, ils tentent de le rejoindre mais ils sont empêchés par les policiers : bien que résidents de la ville, ils sont inconnus des forces de l'ordre. Le chef local intervient alors, affirmant qu'il s'agit bien de membres de son groupe et il permet à ses pairs de le rejoindre en transférant sur eux la légitimité de sa présence en tant que bouffon. Autrement dit, les bouffons étrangers et inconnus s'appuient sur la légitimité et la visibilité des chefs locaux pour accéder à un espace social et cérémoniel, qu'il soit rituel ou séculier.

Ces chefs agissent également en qualité d'intermédiaires et de coordinateurs des festivités. Qu'ils soient les initiateurs d'une cérémonie ou qu'ils relayent l'information, ils doivent s'assurer que les deux parties, organisateurs et korodugaw 
invités, sont satisfaites de l'intervention. D'un côté, les participants étrangers doivent trouver au cours de leur séjour suffisamment de nourriture et de ressources pour compenser les pertes liées à leurs déplacements (frais de transport, arrêt du travail). De l'autre, il importe que le nombre de bouffons actifs participants soit à la mesure des festivités, sans que les hôtes et organisateurs se trouvent en défaut dans leur devoir d'accueil, ou qu'ils soient dépassés par une charge financière et logistique trop lourde (préparation des repas, logements, etc.). Avec ce rôle des chefs locaux, le couple résidents (immobiles)/étrangers (mobiles) donne naissance à une relation entre les invités et leurs hôtes (qu'ils soient korodugaw ou non). Pour les bouffons, cette relation s'étend à l'ensemble du village puisqu'ils ne prennent pas seulement en compte les capacités d'accueil des organisateurs mais également les opportunités offertes au sein du village dans son ensemble, en particulier la tenue d'une foire hebdomadaire qui constitue, pour les bouffons en déplacement, une source de dons.

Enfin, l'ensemble des bouffons résidents est mobilisé au cours des processions bouffonnes qui se déroulent dans la localité. Contrairement aux étrangers qui apparaissent aux villageois sous leur seule identité sociale de koroduga, les bouffons résidents sont avant tout des habitants connus dans leurs multiples identités sociales: membre de telle famille, allié, chef de village, imam, boutiquier, enseignant, celle de bouffon n'étant que secondaire puisqu'elle ne s'exprime que rarement. Connus et respectés par ailleurs, les résidents vont favoriser le consentement des villageois. En effet, pratiquer la bouffonnerie n'est pas aisé car cela se fonde sur un dépassement des limites (gloutonnerie, absence de honte, simulacres de vols) qui doit dans le même temps rester acceptable pour leurs interlocuteurs. Afin d'éviter que la bouffonnerie ne tombe dans le registre de la transgression réelle, la communication avec la cible de leurs jeux doit être maintenue. Dans ce sens, les korodugaw locaux agissent principalement comme guides. Ceux qui accompagnent la procession indiquent les concessions des villageois favorables à la visite des bouffons, évitant ainsi les tensions et les éventuels conflits. Ces guides sont les membres actifs du groupement villageois, en particulier des femmes qui voyagent beaucoup moins que les hommes mais qui néanmoins circulent dans l'enceinte de la localité en venant gonfler le rang des processions de leurs pairs étrangers en visite. Les bouffons inactifs qui ne se déplacent pas (hors ou dans le village) orientent eux aussi le parcours de la procession car ils seront visités en priorité. Ils constituent autant de relais dans le village assurant un minimum de lieux de passage pour les bouffons actifs qui dans ces déplacements orientés en profiteront pour se rendre chez d'autres habitants.

Par cette double qualité de koroduga et résident et parce qu'ils sont connus dans leurs multiples identités sociales, les bouffons locaux permettent aux mobiles de s'inscrire dans l'espace social de la localité visitée. À l'inverse, les mobiles, par leur qualité d'étranger, apparaissent dans leur seule identité de koroduga (même si elle est construite à partir d'autres identités sociales) et permettent à ces locaux de réaffirmer cette même identité.

\section{Circuler pour renforcer l'ancrage local des bouffons}

\section{L'influence de la mobilité spatiale sur la mobilité sociale des bouffons actifs}

Tous les chefs korodugaw n'ont pas la même influence, certaines caractéristiques les distinguant les uns des autres. Leur âge, leurs connaissances ésotériques (médecines, invocations, géomancie, etc.), leurs relations, l'histoire de leur village sont autant de facteurs qui participent à forger une notoriété. Elle se fonde aussi sur les déplacements cérémoniels. Comme pour tout Malien, se rendre aux festivités de 
mariage ou de circoncision de ses proches et de ses clients est une marque et une source de respect qui permet non seulement de renforcer son réseau de relations, mais aussi de s'en créer de nouveaux (Vuarin, 1994). Les bouffons participent en plus à des cérémonies organisées par des personnes avec lesquelles ils n'entretiennent aucune obligation cérémonielle ou relationnelle. Dans ce cas, leur présence vise avant tout à faire valoir leur nom par l'intermédiaire du réseau de korodugaw. Mais ces participations cérémonielles sont fortement contingentes. Soit parce qu'elles sont planifiées par les bouffons à la fin d'une manifestation, lorsqu'avant de repartir chez eux, ils fixent la date de leur prochain rassemblement, soit parce qu'elles sont le fait d'une invitation qui intervient au cours d'un déplacement. Sortir de cette dynamique circulatoire signifie alors sortir de l'accès à l'information et, au fur et à mesure, perdre sa place au sein d'un réseau d'activité de bouffons. Une femme qui avait une position de chef se plaignait ainsi que, ne pouvant plus pratiquer la bouffonnerie pour des raisons de santé, les membres de son groupe d'activité ne venaient même plus la prévenir des manifestations à venir : elle se trouvait ainsi exclue non seulement de sa participation, mais aussi de la prise des décisions.

En renforçant les relations entre les bouffons pratiquants, cette position active dans les déplacements offre également la possibilité d'inviter leurs pairs pour effectuer des festivités dans leurs villages. Être en position de recevoir des étrangers permet tout d'abord au chef de groupe d'activités de choisir la partie de son réseau qu'il veut renforcer. Ainsi, un chef âgé encore actif s'est vu confier des enfants afin qu'ils soient intégrés au korodugaya. Après leur avoir fourni des médecines, il décide d'organiser des baptêmes bouffons. Plusieurs choix s'offraient à lui, comme réunir les femmes korodugaw de son village ou faire appel à ses pairs actifs d'un village voisin avec qui il entretient de nombreuses relations. Mais il a décidé d'inviter des korodugaw résidant dans la ville de Ségou (située non loin de là mais de l'autre côté du fleuve) où vient de se fonder une nouvelle association de bouffons dans laquelle il est déjà engagé. D’un côté, cette invitation lui permet de réaffirmer sa position au sein du réseau nouvellement créé. Tout d'abord en se montrant comme un pourvoyeur de médecines réputé dans son village et dans les environs: tous les bouffons actifs n'ayant pas de connaissances dans ces domaines, ceux qui les maitrisent se placent en effet au sommet de la hiérarchie koroduga, car ils sont à la fois consultés par de nombreux clients, mais aussi par leurs pairs qui souhaitent acquérir certaines médecines pour les redistribuer ensuite. Les bouffons qui se voient confier plusieurs enfants acquièrent une position d'autant plus élevée que la survie des enfants atteste de l'efficacité de leurs pouvoirs dans ce domaine. D'un autre côté, ce chef koroduga participe également à accroître la notoriété des membres de son réseau qu'il a choisi d'inviter. En effet, ceux qui participent aux baptêmes bouffons attribuent chacun leur prénom à l'un des enfants adoptés qui devient alors leur homonyme. Au Mali, la position d'éponyme est d'une manière générale honorifique. Elle l'est de manière différente pour les bouffons, l'énonciation du nombre et du lieu de résidence des homonymes étant aussi une manière d'afficher leur sphère d'influence, leur réputation à partir d'un réseau bouffon. Autrement dit, en invitant certains de ses pairs pour les baptêmes, ce chef koroduga s'assure de leurs reconnaissances à la fois pour ses propres capacités et pour avoir contribué à accroître leurs notoriétés.

Enfin, la circulation renforce également la position du chef local dans son propre village. Les parcours des bouffons sont orientés par la visite des homonymes auxquels ils sont tenus de rendre visite régulièrement. Par conséquent, attribuer le prénom des membres de son réseau d'activité aux enfants qui lui ont été confiés inscrit durablement son village dans les parcours de circulation des bouffons. En 
accueillant ces étrangers, il affirme sa position de koroduga dans l'espace villageois montant qu'il est reconnu dans un réseau plus large et offrant également aux résidents la possibilité d'assister à des festivités et de rencontrer des figures réputées à même de leur fournir des médecines et de répondre à leurs différents besoins. Autrement dit, un koroduga invitant se positionne comme un nœud de réseau entre les villageois et les korodugaw dont le statut d'étranger donne une plus-value aux pouvoirs qui leur sont accordés. La circulation permet aux mobiles étrangers d'élargir leur sphère d'influence, et aux immobiles (temporaires) les recevant de maintenir une position dans le réseau par l'intermédiaire de sa localité. De l'alternance entre ces deux positions émergent certaines figures réputées de la bouffonnerie.

\section{L'intégration des immobiles inactifs dans la bouffonnerie}

Les chefs korodugaw n'ont pas le monopole de l'organisation des cérémonies. Quiconque peut inviter des bouffons, car leur prestation est à la fois festive et propitiatoire. L'invitation s'adresse alors moins à des proches que plus largement aux membres d'une catégorie sociale, celle de koroduga, sans qu'une relation préalable les unisse nécessairement. Ce caractère impersonnel est renforcé par les prestations des bouffons participants qui, contrairement aux animateurs rémunérés et aux invités, n'assistent pas à l'ensemble des festivités : ils arrivent en cours pour effectuer leurs prestations et partent lorsqu'ils le souhaitent. Parmi les organisateurs potentiels figurent des familles korodugaw qui n'ont pas l'habitude de pratiquer la bouffonnerie. Là aussi l'invitation s'adresse à des individus sur la base de leur identité de bouffon, mais elle prend dans ce cas une autre qualité pour les organisateurs, celle de rappeler une catégorie d'appartenance qu'ils n'ont pas souvent l'occasion de mettre en avant. La mobilité des bouffons actifs joue à ce moment-là un rôle important. La pratique de la bouffonnerie étant caractérisée par des danses et des attitudes burlesques, extraverties et qualifiées par l'absence de honte, peu de korodugaw se permettent d'exercer cette activité. En revanche, recevoir chez soi des membres actifs de sa catégorie implique une participation par des danses et des dons, en signe de respect de sa filiation. Qu'ils soient invités ou simplement reçus par des membres inactifs, la circulation des bouffons leur offre l'occasion de manifester cette identité sociale sans qu'ils aient à se déplacer ni même à émettre l'intention de pratiquer la bouffonnerie, c'est-à-dire en gardant la réserve propre à leur position sociale (de femme mariée, de noble, etc.). Autrement dit, la circulation permet de rassembler actifs et inactifs et de réinscrire la pratique de la bouffonnerie dans un espace social où l'attitude de réserve est normalement attendue.

La mobilité des uns permet ainsi d'activer l'identité sociale des autres, non seulement pour les familles korodugaw passives qui invitent des actifs, mais aussi pour tout koroduga se trouvant sur leur passage. Lorsqu'ils marchent en parure et aux sons des instruments de musique dans les rues des villes et des villages, mais aussi lorsqu'ils se reposent dans la concession qui leur est allouée, un certain nombre de personnes réagissent spontanément à leur présence. Certains les saluent, d'autres marquent leur commune appartenance par des dons, d'autres encore donnent à voir des signes caractéristiques de leur identité sociale koroduga: un collier sorti d'une malle de la maison, un comportement qui serait perçu comme déplacé en dehors du cadre de la bouffonnerie. Au cours des processions dans les marchés, sollicitant des dons d'une échoppe à une autre, les commerçants korodugaw se distinguent des autres en interrompant un instant leur commerce pour participer aux danses bouffonnes, parfois à la surprise de leurs voisins car pour certains cette identité n'est pas connue jusqu'à ce qu'ils la manifestent ainsi. Cette activation de l'identité sociale auprès d'individus peu enclins de par leur position ou leur caractère 
à pratiquer la bouffonnerie se donne à voir de manière plus prégnante encore avec des enfants donnés. En effet, la dernière étape du processus d'adoption consiste à se rendre vers l'enfant et à marquer son intégration au groupe corporellement par une participation aux danses. Il n'est pas nécessaire de pratiquer activement la bouffonnerie avec l'intention de se rendre à des cérémonies, mais il n'est pas non plus suffisant de seulement dire, ou savoir, que l'on est koroduga. Il importe de montrer l'acceptation de cette identité en dansant lorsque des bouffons actifs sont de passage dans son village et l'y invitent. La qualité d'étranger de ces mobiles et la dimension impersonnelle $\mathrm{du}$ groupe facilitent cette participation dansée et le dépassement de la honte. Au final, la circulation, en mettant en relation actifs et inactifs, mobiles et immobiles, permet l'expression de l'identité sociale koroduga et sa pérennisation.

\section{Conclusion}

L'étude de l'immobilité dans la circulation, thème des journées qui nous ont réunis à Poitiers, s'applique ici aux membres d'une catégorie sociale acquise par la filiation ou l'adoption. Cette appartenance catégorielle commune est insuffisante pour assurer la visibilité et la pérennité du groupe dans l'espace social, elle doit s'actualiser par l'activité qui le caractérise : la bouffonnerie. Son exercice impliquant une mobilité tend à distinguer différents types de bouffons: les actifs et les inactifs, les mobiles intervillageois ou intravillageois et les immobiles. Mais cette différenciation, loin de diviser le groupe social, participe à sa morphogenèse et à son unité.

Pour analyser ce processus, un troisième terme a été mobilisé, celui d'ancrage local. Souvent associé à l'immobilité (Fol, 2010), ces deux notions ne se confondent pas strictement puisque l'une a pour référence des déplacements tandis que l'autre a pour référence un espace social. Cette distinction est importante dans le cas qui nous préoccupe. D'une part l'ancrage local s'applique aussi bien aux individus qui se déplacent qu'à ceux qui ne se déplacent pas. D'autre part, l'ancrage local a une portée plus large que l'immobilité car il ne concerne pas seulement les individus, il s'applique également aux groupements sociaux et à l'activité de bouffonnerie. De la même manière, la circulation ne se confond pas avec la mobilité des bouffons, en particulier parce qu'elle forge un réseau qui met en relation les différents types de bouffons et de regroupements, mobiles et immobiles. L'articulation entre ancrage local et circulation constitue donc un cadre englobant pour l'analyse qui permet de considérer ensemble ce qui donne forme à la bouffonnerie : les déplacements ainsi que les lieux investis par les korodugaw.

La distinction entre mobiles et immobiles s'avère en revanche utile pour saisir les mécanismes et les effets de cette dynamique globale. Ces deux positions sont relatives, non seulement car elles alternent pour chaque individu, plus encore car elles dépendent de l'échelle spatio-temporelle considérée (intervillageoise ou intravillageoise). Cependant, elles sont bien effectives car la rencontre entre des individus qui se déplacent avec ceux qui ne se déplacent a pour effet de forger des relations complémentaires. Lorsque les bouffons entrent dans un village, la relation se construit autour du couple résidents/étrangers. La particularité des mobiles étrangers est qu'ils se présentent avant tout par leur identité sociale de bouffons alors que ceux qu'ils retrouvent (les « immobiles ») sont des villageois connus dans leurs multiples identités sociales où celle de bouffon n'est que secondaire. Comme on peut s'y attendre, ces derniers favorisent l'insertion des premiers dans leur localité ainsi que l'exercice de la pratique de la bouffonnerie car, du fait de leur double position, ils agissent comme médiateurs dans l'espace villageois. Mais les étrangers favorisent également l'inscription sociale des seconds en tant que koroduga sur leur lieu de 
vie car ils permettent de mettre en avant cette identité sociale par la pratique de la bouffonnerie. Une autre configuration relationnelle apparaît ensuite à l'échelle d'une activité qui se déploie dans l'enceinte villageoise. Ici, le couple mobile/immobile prend la forme d'une relation entre visiteurs et visités, où étrangers et locaux actifs se fondent dans un même groupe processionnel face aux habitants qui les accueillent. Ces processions et ces visites, par les relations de dons et les activités propitiatoires des bouffons qu'elles mettent en œuvre, permettent à la pratique de la bouffonnerie de s'inscrire dans l'espace social villageois.

De ces multiples relations complémentaires se forge une unité du groupe social qui ne dépend plus seulement d'une catégorie d'appartenance commune, en fournissant au groupe social à la fois les conditions de sa cohésion et une position dans l'espace social. La relation visiteur/visité fait le lien entre les bouffons actifs et inactifs et, associée à la qualité d'étranger, elle permet de dépasser les inhibitions qui empêchent les inactifs de manifester leur identité sociale koroduga. De plus, l'association de ces deux relations complémentaires offre aux étrangers l'opportunité d'imposer l'identité sociale koroduga dans l'espace villageois le temps des processions, et de susciter l'adhésion et la participation de l'ensemble des habitants. Ainsi le groupe social ne se définit pas seulement par rapport aux autres. Il se constitue dans un entre-soi fondé sur une identité sociale commune partagée entre mobiles et immobiles et se construit dans la circulation qui met en relation des korodugaw actifs avec des korodugaw inactifs. Ces prestations circulantes valorisent en retour l'identité sociale dans la localité sans que cela passe par l'installation durable de ces membres. Circulation et structure ou organisation stable, loin de s'opposer (Urry, 2000 in Gallez, 2009: 8), sont ici indissociables.

Laure Carbonnel

Doctorante en Anthropologie Université Paris Ouest, - Centre d'Étude des Mondes Africains (UMR CEMAf) Laboratoire d'Ethnologie et de Sociologie Comparative (UMR LESC) laure.carbonnel@gmail.com

\section{Bibliographie}

Colleyn, Jean-Paul (1988) Les chemins de NyaCulte de possession an Mali, Paris, EHESS, $221 \mathrm{p}$.

Colleyn, Jean-Paul (2010) Les Chevaux de la satire. Les Kórèdugaw du Mali, Paris, Gourcuff \& Gradenigo, 159 p.

De Sales, Anne (1995) Chamanes et Bouffons (Népal et Sibérie), Études Mongoles et Sibériennes, Cahier 26, pp. 17-40 (Variations chamaniques 2).

Dieterlen, Germaine ; Cissé, Youssouf Tata (1972) Les fondements de la société d'initiation $d u$ Komo, Paris ; La Haye, Mouton \& Co, 326 p.

Fer, Yannick (2012) Le protestantisme polynésien. De l'église locale aux réseaux évangéliques, Archives des sciences sociales des religions, $\mathrm{n}^{\circ} 157$, pp. 47-66.

Fol, Sylvie (2010) Mobilité et ancrage dans les quartiers pauvres, Regards Sociologiques, $\mathrm{n}^{\circ} 40$, pp. $27-43$.

Gallais, Jean (1962) Signification du groupe ethnique au Mali, L'Homme, vol. 2, $\mathrm{n}^{\circ} 2$, pp. 106-29. 
Gallez, Caroline ; Kaufmann Vincent (2009) Aux racines de la mobilité en sciences sociales: contribution au cadre d'analyse socio-historique de la mobilité quotidienne, in Guigueno, V.; Flonneau, M. (Ed.) De l'bistoire des transports à l'bistoire de la mobilité, Rennes, Presses Universitaires de Rennes, pp. 41-55.

Handelman, Don (1981) The ritual-clown : attribute and affinities, Anthropos, no76 (3/4), pp. 321-70.

Jolly, Eric (1994) Diffusion de trois cultes dans le sud du pays Dogon : juru, ina et aramanu-na, Journal des Africanistes, nº4 (2), pp. 3-38.

Luneau, René (1974) Les chemins de la noce La femme et le mariage dans la société rurale au Mali, Paris, Université René Descartes UER des sciences sociales, $712 \mathrm{p}$. Th. d'État: Ethnologie : Paris : 1974.

Ma Mung, Emmanuel (2009) Le point de vue de l'autonomie dans l'étude des migrations internationales: penser de l'intérieur les phénomènes de la mobilité, in Dureau, F. ; Hilly, M.A. (Ed.) Les mondes de la mobilité, Rennes, Presses Universitaire de Rennes, pp. 25-38.

Quesnel, André (2009) De la communauté territoriale à l'organisation familiale en archipel: la mobilité spatiale des familles rurales en Afrique de l'Ouest et au Mexique, in Dureau, F. ; Hilly, M.A. (Ed.) Les mondes de la mobilité, Rennes, Presses Universitaire de Rennes, pp. 67-103.

Simmel, Georg (2010) Sociologie. Étude sur les formes de la socialisation, Paris, Quadrige/PUF, $756 \mathrm{p}$.

Tarabout, Gilles (1998) Des gags dans le culte. Remarques sur la bouffonnerie rituelle au Kérala. Suivi de Notes sur théâtre et possession ou petite collection d'historiettes, in Bansat-Boudon, L. (Ed.) Théatres indiens, Paris, EHESS, pp. 269-299.

Tarrius, Alain (1993) Territoires circulatoires et espaces urbains. Différenciation des groupes migrants, Annales de la recherche urbaine, n59-60, pp. 51-60.
Vuarin, Robert (1994) L'argent et l'entregent, Cabiers des sciences bumaines, vol. 30 (1-2), pp. 255-273.

Zahan, Dominique (1960) Sociétés d'initiation Bambara. Le n'domo, le korè, Paris ; La Haye, Mouton \& Co., 438 p. 